\title{
A New Intelligent Method for Eliminating Unknown (Nuisance) Parameters from Underlying Models as an Alternative to the Bayesian Approach
}

\author{
N. A. Nechval, ${ }^{1, *}$, G. Berzins ${ }^{1}$, K. N. Nechval ${ }^{2}$, Zh. Tsaurkubule ${ }^{3}$, M. Moldovan ${ }^{4}$ \\ ${ }^{1}$ BVEF Research Institute, University of Latvia, Riga, 1586, Latvia. \\ ${ }^{2}$ Department of Aviation, Transport and Telecommunication Institute, Riga, 1019, Latvia. \\ ${ }^{3}$ Department of Statistics, Baltic International Academy, Riga, 1019, Latvia. \\ ${ }^{4}$ Department of Biometry, University of Adelaide, Adelaide, 5005, state of South Australia, Australia.
}

\begin{abstract}
How to cite this paper: N. A. Nechval, G. Berzins, K. N. Nechval, Zh. Tsaurkubule, M. Moldovan. (2022) A New Intelligent Method for Eliminating Unknown (Nuisance) Parameters from Underlying Models as an Alternative to the Bayesian Approach. Journal of Applied Mathematics and Computation, 6(1), 53-65.

DOI: $10.26855 /$ jamc.2022.03.008
\end{abstract}

Received: December 29, 2021

Accepted: January 24, 2022

Published: March 1, 2022

*Corresponding author: N. A. Nechval, BVEF Research Institute, University of Latvia, Riga, 1586, Latvia.

Email:nechval@telenet.lv

\begin{abstract}
The big question in statistics is: How can we eliminate the unknown (nuisance) parameter from an underlying model? Eliminating unknown (nuisance) parameters from an underlying model is universally recognized as a major problem of statistics and has been formally studied in virtually all approaches to inference. A surprisingly large number of elimination methods have been proposed in the literature on this topic. The classical method of elimination of unknown (nuisance) parameters from the model, which is used repeatedly in the large sample theory of statistics, is to replace the unknown (nuisance) parameter by an estimated value. However, this method is not efficient when dealing with small data samples. The Bayesian approach is dependent of the choice of priors. In this paper, a new method is proposed to eliminate the unknown (nuisance) parameter from the underlying model. This method isolates and eliminates unknown (nuisance) parameters from the underlying model as efficiently as possible. Unlike the Bayesian approach, the proposed method is independent of the choice of priors and represents a novelty in the theory of statistical decisions. It allows one to eliminate unknown parameters from the problem and to find the efficient statistical decision rules, which often have smaller risk than any of the well-known decision rules. To illustrate the proposed method, some practical applications are given.
\end{abstract}

\section{Keywords}

Underlying model, Parametric uncertainty, Elimination of unknown (nuisance) parameters, Efficient statistical decisions

\section{Introduction}

The problem of determining an optimal decision rule in the absence of complete information about the underlying distribution, i.e., when we specify only the functional form of the distribution and leave some or all of its parameters unspecified, is seen to be a standard problem of statistical estimation. Unfortunately, the classical theory of statistical estimation has little to offer in general type of situation of loss functions. Eliminating unknown (nuisance) parameters from an underlying model is widely recognized as one of the main problems of statistics. A surprisingly large number of elimination methods have been proposed by various authors on this topic. The big question in statistics is: How can we exclude a nuisance (unknown) parameter from an underlying model? An incredible amount of effort and ingenuity has been ex- 
pended in finding reasonable answers to this question [1, 2]. In this paper, an innovative statistical pivot-based approach to elimination of unknown (nuisance) parameters from the underlying models is proposed for constructing efficient statistical decisions under parametric uncertainty. The main aim of the present paper is to show how the innovative statistical pivot-based approach may be employed in the particular case of constructing the exact statistical prediction limits and shortest-length or equal tails confidence intervals for future random quantities under parametric uncertainty. The approach used here is a special case of more general considerations applicable whenever the statistical problem is invariant under a group of transformations, which acts transitively on the parameter space [3-18].

\section{Statistical Prediction of Future Random Quantities}

Statistical prediction of future random quantities (future outcomes, order statistics, etc.) based on the past and current data is the most prevalent form of statistical inference. Predictive inferences for future random quantities are widely used in risk management, finance, insurance, economics, hydrology, material sciences, telecommunications, and many other industries. Predictive inferences(predictive distributions, prediction limits, confidence (prediction) intervals) for future random quantities on the basis of the past and present knowledge represent a fundamental problem of statistics, arising in many contexts and producing varied solutions. Statistical prediction is the process by which values for unknown observables (potential observations yet to be made or past ones which are no longer available) are inferred based on current observations and other information at hand.

\section{Types of Statistical Prediction Problems}

There are the following types of prediction problems:

A. New-sample prediction problem. In this case, the data from a past sample of size $n$ are used to make prediction on one or more future units in a second sample of size $m$ from the same process or population. For example, based on previous (possibly censored) life test data, one could be interested in predicting the following: (1) time to failure of a new item $(m=1)$; (2) time until the $k$ th failure in a future sample of $m$ units, $m \geq k$; (3) number of failures by time $\tau^{\bullet}$ in a future sample of $m$ units. Formally, we call the problems in this category as two-sample problems.

B. Within-sample prediction problem. In this case, the problem is to predict future events in a sample or process based on the early-failure data from that sample or process. For example, if $n$ units are followed until censoring time $\tau_{c}$ and there are $r$ observed ordered failure times, $X_{1} \leq \ldots \leq X_{r}$, one could be interested in predicting the following: 1) time of next failure; 2) time until $l$ additional failures, $l \leq n-r$; 3) number of additional failures in a future interval $\left(\tau_{c}, \tau^{\bullet}\right)$. Formally we call the problems in this category as one-sample problems.

C. New-within-sample prediction problem. In this case, the problem is to predict future events in a sample or process based on both the early-failure data from that sample or process and the data from a previous sample (i.e., when for predicting a future failure time of a unit in a new sample there are available both the early-failure data from that sample and the data from a previous sample). Formally we call the problems in this category as multi-sample problems.

In this paper, the within-sample prediction problems are considered.

\section{Types of Statistical Prediction Limits}

Usually, two types of statistical prediction limits are defined: i) statistical $\gamma$-content prediction limits with expected $(1-\alpha)$-confidence on future outcomes, ii) statistical prediction limits with expected $(1-\alpha)$-confidence on future outcomes (or simply $(1-\alpha)$-prediction limits). The statistical $\gamma$-content prediction limit with expected $(1-\alpha)$-confidence seems to be more useful than the statistical prediction limit with expected $(1-\alpha)$-confidence but is relatively difficult to construct.

\section{Pivot-Based Method for Eliminating Unknown (Nuisance) Parameters from Underlying Models}

The pivot-based method for eliminating unknown (nuisance) parameters from the underlying model includes the following 3 stages:

Stage 1 (Invariant statistical embedding of a sample statistic in the underlying model to construct a pivotal quantity and isolate unknown (nuisance) parameter from the problem). At this stage, an invariant embedding of a sample statistic in the underlying model (say, probability distribution, decision criterion (performance index), etc.) is carried out to construct a pivotal quantity (or simply a pivot) in order to isolate the unknown (nuisance) parameter from the problem, since the pivot's probability distribution does not depend on the unknown parameter. 
Stage 2 (Averaging the underlying model over the probability distribution of the pivotal quantity to eliminate unknown (nuisance) parameter from the problem). At this stage, the underlying model (say, probability distribution, decision criterion (performance index), etc.) is averaged over the probability distribution of the pivotal quantity in order to eliminate the unknown (nuisance) parameter from the problem.

Stage 3 (Efficient decision-making process). At this stage, when the unknown (nuisance) parameter has been eliminated from the underlying model (say, probability distribution, decision criterion (performance index), etc.), it can be found an efficient statistical decision rule.

\section{Exponential Distribution}

The one-parameter exponential distribution is one of the most significant and widely used distribution in statistical practice. It possesses several important statistical properties, and yet exhibits great mathematical tractability. This distribution is one of the most commonly used models in life-testing and reliability studies.

Let $\mathbf{Y}=\left(Y_{1} \leq \ldots \leq Y_{k}\right)$ be the first $k$ ordered observations (order statistics) in a sample of size $m$ from the exponential distribution with the probability density function

$$
f_{\beta}(y)=\beta^{-1} \exp (-y / \beta), \quad \beta>0, y>0,
$$

and the cumulative distribution function

$$
F_{\beta}(y)=1-\exp (-y / \beta),
$$

where $\beta$ is the scale parameter. It is assumed that the parameter $\beta$ is unknown. In Type II censoring, which is of primary interest here, the number of survivors is fixed and $Y_{k}$ is a random variable. The MLE's $\hat{\beta}$ of the parameter $\beta$ is given by

$$
\widehat{\beta}=\frac{S_{k}}{k}=\frac{\sum_{i=1}^{k} Y_{i}+(m-k) Y_{k}}{k},
$$

It is known that $S_{k}$ is the complete sufficient statistic for $\beta$. Then

$$
V_{k}=S_{k} / \beta
$$

is the pivotal quantity, the probability density function of which is given by

$$
f\left(v_{k} \mid k\right)=\frac{1}{\Gamma(k)} v_{k}^{k-1} \exp \left(-v_{k}\right), \quad v_{k} \geq 0 .
$$

The probability density function of the order statistic $Y_{k}$ is given by

$$
\begin{array}{r}
g_{\beta}\left(y_{k}\right)=\frac{m !}{(k-1) !(m-k) !}\left[F_{\beta}\left(y_{k}\right)\right]^{k-1}\left[1-F_{\beta}\left(y_{k}\right)\right]^{m-k} f_{\beta}\left(y_{k}\right)=\frac{1}{\mathrm{~B}(k, m-k+1)} \sum_{j=0}^{k-1}\left(\begin{array}{c}
k-1 \\
j
\end{array}\right) \\
\times(-1)^{j} \exp \left(-\frac{y_{k}}{\beta}(m-k+1+j)\right) \frac{1}{\beta}, \quad y_{k} \in(0, \infty) .
\end{array}
$$

The cumulative distribution function of the $k$ th order statistic $Y_{k}$ is given by

$$
G_{\beta}\left(y_{k}\right)=\operatorname{Pr}\left(Y_{k} \leq y_{k}\right)=\int_{0}^{y_{k}} g_{\beta}(y) d y=1-\frac{1}{\mathrm{~B}(k, m-k+1)} \sum_{j=0}^{k-1}\left(\begin{array}{c}
k-1 \\
j
\end{array}\right)(-1)^{j} \frac{\exp \left[-\frac{y_{k}}{\beta}(m-k+j+1)\right]}{m-k+j+1} .
$$

It follows from (7) that the cumulative distribution function of the pivotal quantity

is given by

$$
V_{k}^{\cdot}=\frac{Y_{k}}{\beta}
$$




$$
G\left(v_{k}^{*}\right)=1-\frac{1}{\mathrm{~B}(k, m-k+1)} \sum_{j=0}^{k-1}\left(\begin{array}{c}
k-1 \\
j
\end{array}\right)(-1)^{j} \frac{\exp \left[-v_{k}^{\bullet}(m-k+j+1)\right]}{m-k+j+1} .
$$

The probability density function of $V_{k}^{\bullet}$ is given by

$$
g\left(v_{k}^{\cdot}\right)=\frac{d G\left(v_{k}^{\cdot}\right)}{d v_{k}^{*}}=\frac{1}{\mathrm{~B}(k, m-k+1)} \sum_{j=0}^{k-1}\left(\begin{array}{c}
k-1 \\
j
\end{array}\right) \times(-1)^{j} \exp \left(-v_{k}^{\cdot}(m-k+1+j), \quad v_{k}^{\cdot} \in(0, \infty) .\right.
$$

Theorem 1 (Conditional probability distribution of the lth order statistic). Let $Y_{1} \leq \ldots \leq Y_{k}$ be the first $k$ ordered observations (order statistics) in a sample of size $m$ from a continuous distribution (say, exponential) with some probability density function $f_{\beta}(y)$ and distribution function $F_{\beta}(y)$, where $\beta$ is a parameter. Then the conditional probability distribution function of $Y_{l}(1 \leq k<l \leq m)$ given $Y_{k}=y_{k}$ is determined as

$$
\begin{aligned}
\operatorname{Pr}\left(Y_{l} \leq y_{l} \mid Y_{1}=y_{1}, \ldots, Y_{k}=y_{k}\right)=\operatorname{Pr}\left(Y_{l} \leq y_{l} \mid Y_{k}=y_{k}\right)=1-\frac{1}{\mathrm{~B}(l-k, m-l+1)} \sum_{j=0}^{l-k-1}\left(\begin{array}{c}
l-k-1 \\
j
\end{array}\right) \frac{(-1)^{j}}{m-l+1+j}\left[\frac{\bar{F}_{\beta}\left(y_{l}\right)}{\bar{F}_{\beta}\left(y_{k}\right)}\right]^{m-l+1+j} \\
=1-\frac{1}{\mathrm{~B}(l-k, m-l+1)} \sum_{j=0}^{l-k-1}\left(\begin{array}{c}
l-k-1 \\
j
\end{array}\right) \frac{(-1)^{j}}{m-l+1+j} \times \exp \left(-\frac{y_{l}-y_{k}}{\beta}(m-l+1+j)\right)=G_{\beta}\left(y_{l} \mid y_{k}\right) .
\end{aligned}
$$

where

$$
\bar{F}_{\beta}(y)=1-F_{\beta}(y) .
$$

Proof. The proof is omitted here and will appear elsewhere.

It follows from (11) that

$$
\begin{aligned}
\operatorname{Pr}\left(Y_{l}>y_{l} \mid Y_{k}=y_{k}\right)=1-\operatorname{Pr}\left(Y_{l} \leq y_{l} \mid Y_{k}=y_{k}\right) & =\frac{1}{\mathrm{~B}(l-k, m-l+1)} \sum_{j=0}^{l-k-1}\left(\begin{array}{c}
l-k-1 \\
j
\end{array}\right) \frac{(-1)^{j}}{m-l+1+j} \\
& \times \exp \left(-\frac{y_{l}-y_{k}}{\beta}(m-l+1+j)\right)=\bar{G}_{\beta}\left(y_{l} \mid y_{k}\right) .
\end{aligned}
$$

It follows from (11) that the cumulative distribution function of a pivotal quantity

$$
V_{l k}=\frac{Y_{l}-Y_{k}}{\beta}
$$

is given by

$$
G\left(v_{l k}\right)=1-\frac{1}{\mathrm{~B}(l-k, m-l+1)} \sum_{j=0}^{l-k-1}\left(\begin{array}{c}
l-k-1 \\
j
\end{array}\right) \frac{(-1)^{j}}{m-l+1+j} \exp \left(-v_{l k}(m-l+1+j)\right) .
$$

The probability density function of the pivotal quantity $V_{l k}$ is given by

$$
g\left(v_{l k}\right)=\frac{d G\left(v_{l k}\right)}{d v_{l k}}=\frac{1}{\mathrm{~B}(l-k, m-l+1)} \sum_{j=0}^{l-k-1}\left(\begin{array}{c}
l-k-1 \\
j
\end{array}\right)(-1)^{j} \exp \left(-v_{l k}(m-l+1+j)\right), \quad v_{l k} \geq 0 .
$$

\section{Statistical Pivot-Based Estimation of Probability Distributions}

Suppose $Y$ is a future observation from the same distribution (1), independent of $\mathbf{Y}=\left(Y_{1} \leq \ldots \leq Y_{k}\right)$. Then the pivot-based estimate of (2)can be determined as follows:

Step 1. Invariant embedding of $S_{k}$ in (2) to isolate unknown parameter $\beta$ from the problem through $V_{k}$,

$$
F_{\beta}(y)=1-\exp (-y / \beta)=1-\exp \left(-\frac{y}{s_{k}} \frac{s_{k}}{\beta}\right)=1-\exp \left(-\frac{y}{s_{k}} v_{k}\right)=F_{\beta}\left(\frac{y}{s_{k}} v_{k}\right),
$$

where 


$$
W=\frac{Y}{S_{k}}
$$

is an ancillary statistic.

Step 2. Averaging (17) over the probability distribution of the pivotal quantity $V_{k}$ to eliminate unknown parameter $\beta$ from the problem. It follows from (5) and (17)that the pivot-based estimate of the cumulative distribution function (2) (through the pivot-based approach) is given by

$$
F_{s_{k}}(y)=\int_{0}^{\infty} F_{\beta}\left(\frac{y}{s_{k}} v_{k}\right) f\left(v_{k} \mid k\right) d v_{k}=\int_{0}^{\infty}\left[1-\exp \left(-\frac{y}{s_{k}} v_{k}\right)\right] \frac{1}{\Gamma(k)} v_{k}^{k-1} \exp \left(-v_{k}\right) d v_{k}=1-\frac{1}{\left(1+\frac{y}{s_{k}}\right)^{k}} .
$$

The pivot-based estimate of the probability density function (1) is given by

$$
f_{s_{k}}(y)=\frac{d F_{s_{k}}(y)}{d y}=\frac{k}{s_{k}}\left(1+\frac{y}{s_{k}}\right)^{-k-1}, y \geq 0,
$$

and

$$
\bar{F}_{s_{k}}(y)=1-F_{s_{k}}(y)=\left(1+\frac{y}{s_{k}}\right)^{-k} .
$$

It follows from (19) that the cumulative distribution function of the ancillary statistic (18) is given by

$$
F(w)=1-\frac{1}{(1+w)^{k}} .
$$

The probability density function of the ancillary statistic (18) is given by

$$
f(w)=\frac{d F(w)}{d w}=\frac{k}{(1+w)^{k+1}}, \quad w \geq 0 .
$$

It follows from (5) and (7) that the pivot-based estimate of the cumulative distribution function of the $k$ th order statistic $Y_{k}$ is given by

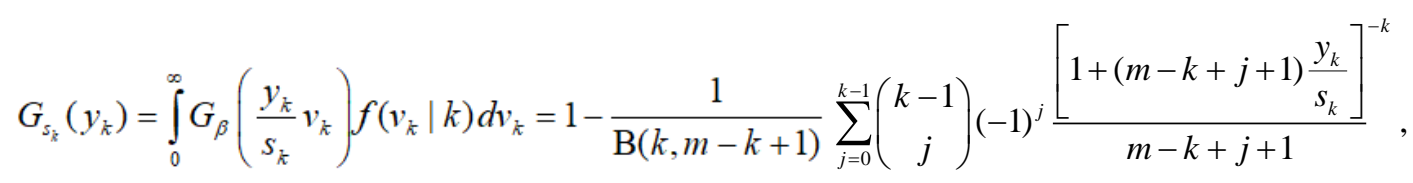

where

$$
W_{k}=\frac{Y_{k}}{S_{k}}
$$

is an ancillary statistic. It follows from (24) that

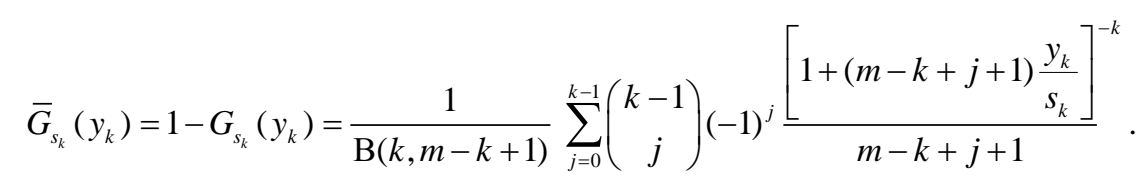

It follows from (24) that the cumulative distribution function of the ancillary statistic $W_{k}$ is given by

$$
G\left(w_{k}\right)=1-\frac{1}{\mathrm{~B}(k, m-k+1)} \sum_{j=0}^{k-1}\left(\begin{array}{c}
k-1 \\
j
\end{array}\right)(-1)^{j} \frac{\left[1+(m-k+j+1) w_{k}\right]^{-k}}{m-k+j+1},
$$

The probability density function of the ancillary statistic $W_{k}$ is given by 


$$
g\left(w_{k}\right)=\frac{d G\left(w_{k}\right)}{d w_{k}}=\frac{1}{\mathrm{~B}(k, m-k+1)} \sum_{j=0}^{k-1}\left(\begin{array}{c}
k-1 \\
j
\end{array}\right)(-1)^{j} k\left[1+(m-k+j+1) w_{k}\right]^{-k-1}, \quad w_{k} \geq 0 .
$$

It follows from (5) and (11) that the pivot-based estimate of the conditional probability distribution function of the lth order statistic $Y_{l}(1 \leq k<l \leq m)$ given $Y_{k}=y_{k}$ is determined (by using the pivot-based method for elimination of the unknown parameter $\beta$ from (11) via $S_{k}=s_{k}$ ) as

$$
G_{s_{k}}\left(y_{l} \mid y_{k}\right)=1-\frac{1}{\mathrm{~B}(l-k, m-l+1)} \sum_{j=0}^{l-k-1}\left(\begin{array}{c}
l-k-1 \\
j
\end{array}\right)(-1)^{j} \frac{\left[\begin{array}{c}
1+(m-l+1+j) \frac{y_{l}-y_{k}}{s_{k}} \\
]^{-k}
\end{array}\right.}{m-l+1+j},
$$

where

$$
W_{l k}=\frac{Y_{l}-Y_{k}}{S_{k}}
$$

is an ancillary statistic. It follows from (29) that

$$
\bar{G}_{s_{k}}\left(y_{l} \mid y_{k}\right)=\frac{1}{\mathrm{~B}(l-k, m-l+1)} \sum_{j=0}^{l-k-1}\left(\begin{array}{c}
l-k-1 \\
j
\end{array}\right)(-1)^{j} \frac{\left[1+(m-l+1+j) \frac{y_{l}-y_{k}}{s_{k}}\right]^{-k}}{m-l+1+j},
$$

It follows from (29) that the cumulative distribution function of the ancillary statistic $W_{l k}$ is given by

$$
G\left(w_{l k}\right)=1-\frac{1}{\mathrm{~B}(l-k, m-l+1)} \sum_{j=0}^{l-k-1}\left(\begin{array}{c}
l-k-1 \\
j
\end{array}\right)(-1)^{j} \frac{\left[1+(m-l+1+j) w_{l k}\right]^{-k}}{m-l+1+j},
$$

The probability density function of the ancillary statistic $W_{l k}$ is given by

$$
g\left(w_{l k}\right)=\frac{d G\left(w_{l k}\right)}{d w_{l k}}=\frac{1}{\mathrm{~B}(l-k, m-l+1)} \sum_{j=0}^{l-k-1}\left(\begin{array}{c}
l-k-1 \\
j
\end{array}\right)(-1)^{j} k\left[1+(m-l+1+j) w_{l k}\right]^{-k-1}, \quad w_{l k} \geq 0 .
$$

It follows from (11) that the conditional probability distribution function of the lth order statistic $Y_{l}(1 \leq k<l \leq m)$ given $Y_{k}=y_{k}$ can be presented as

$$
G_{\beta}\left(y_{l} \mid y_{k}\right)=1-\frac{1}{\mathrm{~B}(l-k, m-l+1)} \sum_{j=0}^{l-k-1}\left(\begin{array}{c}
l-k-1 \\
j
\end{array}\right) \frac{(-1)^{j}}{m-l+1+j} \exp \left(-\frac{y_{l}-y_{k}}{y_{k}}(m-l+1+j) \frac{y_{k}}{\beta}\right),
$$

where

$$
W_{l k}^{\cdot}=\frac{Y_{l}-Y_{k}}{Y_{k}}
$$

is an ancillary statistic. It follows from (10)and (34) that the pivot-based estimate of the conditional cumulative distribution function of the lth order statistic $Y_{l}(1 \leq k<l \leq m)$ given $Y_{k}=y_{k}$ is determined (by using the pivot-based method for elimination of the unknown parameter $\beta$ from (34) via $Y_{k}=y_{k}$ ) as

$$
G_{y_{k}}\left(y_{l} \mid y_{k}\right)=1-\frac{m !}{(m-l) !(l-k-1) !(k-1) !} \sum_{j=0}^{l-k-1}\left(\begin{array}{c}
l-k-1 \\
j
\end{array}\right) \frac{(-1)^{j}}{m-l+1+j} \sum_{i=0}^{k-1}\left(\begin{array}{c}
k-1 \\
i
\end{array}\right)(-1)^{i} \frac{1}{\frac{y_{l}-y_{k}}{y_{k}}(m-l+1+j)+m-k+1+i} \text {. }
$$

Using the identity

$$
\sum_{i=0}^{k-1}\left(\begin{array}{c}
k-1 \\
i
\end{array}\right)(-1)^{i} \frac{1}{\frac{y_{l}-y_{k}}{y_{k}}(m-l+1+j)+m-k+1+i}=\frac{k-1) !}{\prod_{i=0}^{k-1}\left(\frac{y_{l}-y_{k}}{y_{k}}(m-l+1+j)+m-k+1+i\right)},
$$


it follows from (36) that

$$
G_{y_{k}}\left(y_{l} \mid y_{k}\right)=1-\frac{m !}{(m-l) !(l-k-1) !} \sum_{j=0}^{l-k-1}\left(\begin{array}{c}
l-k-1 \\
j
\end{array}\right) \frac{(-1)^{j}}{m-l+1+j}\left[\prod_{i=0}^{k-1}\left(\frac{y_{l}-y_{k}}{y_{k}}(m-l+1+j)+m-k+1+i\right)\right]^{-1}
$$

and

$$
\bar{G}_{y_{k}}\left(y_{l} \mid y_{k}\right)=1-G_{y_{k}}\left(y_{l} \mid y_{k}\right)=\frac{m !}{(m-l) !(l-k-1) !} \sum_{j=0}^{l-k-1}\left(\begin{array}{c}
l-k-1 \\
j
\end{array}\right) \frac{(-1)^{j}}{m-l+1+j}\left[\prod_{i=0}^{k-1}\left(\frac{y_{l}-y_{k}}{y_{k}}(m-l+1+j)+m-k+1+i\right)\right]^{-1} .
$$

It follows from (36) that the cumulative distribution function of the ancillary statistic $W_{l k}^{\bullet}$ is given by

$$
G\left(w_{l k}^{\cdot}\right)=1-\frac{m !}{(m-l) !(l-k-1) !(k-1) !} \sum_{j=0}^{l-k-1}\left(\begin{array}{c}
l-k-1 \\
j
\end{array}\right) \frac{(-1)^{j}}{m-l+1+j} \sum_{i=0}^{k-1}\left(\begin{array}{c}
k-1 \\
i
\end{array}\right)(-1)^{i} \frac{1}{w_{l k}^{\cdot}(m-l+1+j)+m-k+1+i} .
$$

The probability density function of the ancillary statistic $W_{l k}^{\bullet}$ is given by

$$
g\left(w_{l k}^{\cdot}\right)=\frac{d G\left(w_{l k}^{\cdot}\right)}{d w_{l k}^{*}}=\frac{m !}{(m-l) !(l-k-1) !(k-1) !} \sum_{j=0}^{l-k-1}\left(\begin{array}{c}
l-k-1 \\
j
\end{array}\right)(-1)^{j} \sum_{i=0}^{k-1}\left(\begin{array}{c}
k-1 \\
i
\end{array}\right) \frac{(-1)^{i}}{\left[w_{l k}^{\cdot}(m-l+1+j)+m-k+1+i\right]^{2}}, \quad w_{l k}^{\cdot} \in(0, \infty) .
$$

Using the identity (37), it follows from (40)that

$$
G\left(w_{l k}^{\cdot}\right)=1-\frac{m !}{(m-l) !(l-k-1) !} \sum_{j=0}^{l-k-1}\left(\begin{array}{c}
l-k-1 \\
j
\end{array}\right) \frac{(-1)^{j}}{m-l+1+j}\left[\prod_{i=0}^{k-1}\left(w_{k}^{\cdot}(m-l+1+j)+m-k+1+i\right)\right]^{-1}
$$

and

$$
\bar{G}\left(w_{l k}^{\cdot}\right)=1-G\left(w_{l k}^{\cdot}\right)=\frac{m !}{(m-l) !(l-k-1) !} \sum_{j=0}^{l-k-1}\left(\begin{array}{c}
l-k-1 \\
j
\end{array}\right) \frac{(-1)^{j}}{m-l+1+j}\left[\prod_{i=0}^{k-1}\left(w_{k}^{\cdot}(m-l+1+j)+m-k+1+i\right)\right]^{-1} .
$$

\section{Constructing Exact Pivot-Based Prediction Limits}

The following theorems illustrate the pivot-based approach to constructing exact within-sample prediction limits.

Theorem 2 (Exact lower statistical within-sample (1- $\alpha$ ) - prediction limit based on $Y_{k}$ and $S_{k}$ ). Let $Y_{1} \leq \ldots \leq Y_{k}$ be the first $k$ ordered observations (order statistics) in a sample of size $m$ from the exponential distribution with the probability density function(1) and cumulative distribution function (2).It is assumed that the parameter $\beta$ is unknown. In Type II censoring, which is of primary interest here, the number of survivors is fixed and $Y_{k}$ is a random variable. The exact lower statistical within-sample prediction limit with expected (1- $\alpha)$-confidence, $L_{l}^{*} \equiv L_{l}^{*}\left(Y_{k}, S_{k}\right)$, on future outcomes of the lth order statistic $Y_{l}$ from a set of $m$ future ordered observations $Y_{k+1} \leq \ldots \leq Y_{m}$ also from the distribution (1), which satisfies

$$
E_{\beta}\left\{\operatorname{Pr}\left(Y_{l}>L_{l}^{\bullet} \mid Y_{k}=y_{k}\right)\right\}=E_{\beta}\left\{\int_{L_{i}}^{\infty} g_{\beta}\left(y_{l} \mid y_{k}\right) d y_{l}\right\}=E_{\beta}\left\{\bar{G}_{\beta}\left(L_{l}^{*} \mid y_{k}\right)\right\}=\bar{G}_{s_{k}}\left(L_{l}^{*} \mid y_{k}\right)=1-\alpha
$$

is given by

$$
L_{l}^{\cdot}=Y_{k}+\vartheta_{1-\alpha} S_{k}
$$

where 


$$
\vartheta_{1-\alpha}=\arg \left(\frac{\sum_{j=0}^{l-k-1}\left(\begin{array}{c}
l-k-1 \\
j
\end{array}\right) \frac{(-1)^{j}}{m-l+1+j}}{\mathrm{~B}(l-k, m-l+1)}\left[1+(m-l+1+j) \vartheta_{1-\alpha}\right]^{-k}=1-\alpha\right) .
$$

Proof. Assuming that $y_{l}=L_{l}^{*}$, it follows from (31) and (44) that

$$
\bar{G}_{s_{k}}\left(L_{l}^{\cdot} \mid y_{k}\right)=\frac{1}{\mathrm{~B}(l-k, m-l+1)} \sum_{j=0}^{l-k-1}\left(\begin{array}{c}
l-k-1 \\
j
\end{array}\right) \frac{(-1)^{j}}{m-l+1+j}\left[1+(m-l+1+j) \vartheta_{1-\alpha}\right]^{-k}=1-\alpha,
$$

where

$$
\vartheta_{1-\alpha}=\frac{L_{l}^{\bullet}-Y_{k}}{S_{k}}
$$

Then (45) follows from (48). This ends the proof.

Corollary 2.1. The exact upper statistical within-sample (1- $\alpha$ ) - prediction limit $U_{i}^{\bullet}$ on future outcomes of the lth order statistic $Y_{l}$ from a set of $m$ future ordered observations $Y_{k+1} \leq \ldots \leq Y_{m}$ also from the distribution (1), which satisfies

$$
E_{\beta}\left\{\operatorname{Pr}\left(Y_{l} \leq U_{l}^{\bullet} \mid Y_{k}=y_{k}\right)\right\}=E_{\beta}\left\{\int_{0}^{U_{i}^{*}} g_{\beta}\left(y_{l} \mid y_{k}\right) d y_{l}\right\}=E_{\beta}\left\{G_{\beta}\left(U_{l}^{\bullet} \mid y_{k}\right)\right\}=G_{s_{k}}\left(U_{l}^{\bullet} \mid y_{k}\right)=1-\alpha,
$$

is given by

$$
U_{i}^{\bullet}=Y_{k}+\vartheta_{\alpha} S_{k} \text {, }
$$

where $\vartheta_{\alpha}$ is determined by

$$
\vartheta_{\alpha}=\arg \left(\frac{1}{\mathrm{~B}(l-k, m-l+1)} \sum_{j=0}^{l-k-1}\left(\begin{array}{c}
l-k-1 \\
j
\end{array}\right) \frac{(-1)^{j}}{m-l+1+j}\left[1+(m-l+1+j) \vartheta_{\alpha}\right]^{-k}=\alpha\right) .
$$

Theorem 3 (Exact lower statistical within-sample (1- $\alpha$ ) - prediction limit based on $Y_{k}$ ). Let us assume that the first $k-1$ ordered observations (order statistics) $Y_{1} \leq \ldots \leq Y_{k-1}$ in a sample of size $m$ from the exponential distribution with the probability density function (1) are unknown. Then the exact lower statistical within-sample prediction limit with expected (1- $\alpha$ )-confidence, $L_{l}^{*} \equiv L_{l}^{*}\left(Y_{k}\right)$, on future outcomes of the lth order statistic $Y_{l}$ from a set of $m$ future ordered observations $Y_{k+1} \leq \ldots \leq Y_{m}$ also from the distribution (1), which satisfies (44), is given by

$$
L_{l}^{\bullet}=\left(1+\vartheta_{1-\alpha}\right) Y_{k},
$$

where $\vartheta_{1-\alpha}$ is determined by

$$
\vartheta_{1-\alpha}=\arg \left(\frac{m !}{(m-l) !(l-k-1) !} \sum_{j=0}^{l-k-1}\left(\begin{array}{c}
l-k-1 \\
j
\end{array}\right) \frac{(-1)^{j}}{m-l+1+j}\left[\prod_{i=0}^{k-1}\left(\vartheta_{1-\alpha}(m-l+1+j)+m-k+1+i\right)\right]^{-1}=1-\alpha .\right) .
$$

Proof. Assuming that $y_{l}=L_{l}^{*}$, it follows from (42) and (49) that

$$
\bar{G}_{y_{k}}\left(L_{l} \mid y_{k}\right)=\frac{m !}{(m-l) !(l-k-1) !} \sum_{j=0}^{l-k-1}\left(\begin{array}{c}
l-k-1 \\
j
\end{array}\right) \frac{(-1)^{j}}{m-l+1+j} \times\left[\prod_{i=0}^{k-1}\left(\vartheta_{1-\alpha}(m-l+1+j)+m-k+1+i\right)\right]^{-1}=1-\alpha .
$$

where

$$
\vartheta_{1-\alpha}=\frac{L_{l}-Y_{k}}{Y_{k}}
$$


Then (52) follows from (55). This ends the proof.

Corollary 3.1. The exact upper statistical within-sample (1- $\alpha$ ) - prediction limit $U_{l}^{\bullet}$ on future outcomes of the lth order statistic $Y_{l}$ from a set of $m$ future ordered observations $Y_{k+1} \leq \ldots \leq Y_{m}$ also from the distribution (1), which satisfies (49), is given by

$$
U_{i}^{\bullet}=\left(1+\vartheta_{\alpha}\right) Y_{k},
$$

where $\vartheta_{\alpha}$ is determined by

$$
\vartheta_{\alpha}=\arg \left(\frac{m !}{(m-l) !(l-k-1) !} \sum_{j=0}^{l-k-1}\left(\begin{array}{c}
l-k-1 \\
j
\end{array}\right) \frac{(-1)^{j}}{m-l+1+j}\left[\prod_{i=0}^{k-1}\left(\vartheta_{\alpha}(m-l+1+j)+m-k+1+i\right)\right]^{-1}=\alpha .\right) .
$$

\section{Constructing Shortest Length and/or Equal Tails Confidence Intervals for Future Outcomes}

Let us assume that $Y_{1} \leq \ldots \leq Y_{k}$ are the first $k$ ordered observations from the previous random sample of size $m$ from the exponential distribution (1) (the Type II censored sample and the parameter $\beta$ is unknown (nuisance)). What is the $100(1-\alpha) \%$ shortest-length confidence interval for a single future observation from a previously sampled population/process (motivated by a customer's request for an interval to contain the life of a purchased product)?

Answer: To illustrate new-sample prediction via the shortest-length confidence interval, we show how to construct the $100(1-\alpha) \%$ shortest-length confidence interval for a single future observation $Y$ from the same distribution (1).

The random variable (ancillary statistic) $W=Y / S_{k}$ has the cumulative distribution function (22) and probability density function (23). Using $W$, we can construct a $100(1-\alpha) \%$ confidence interval for $Y$ from

$$
P\left(w_{1} \leq W \leq w_{2}\right)=P\left(w_{1} \leq \frac{Y}{S_{k}} \leq w_{2}\right)=P\left(w_{1} S_{k} \leq Y \leq w_{2} S_{k}\right)=1-\alpha
$$

by suitably choosing the decision variables $w_{1}$ and $w_{2}$. Hence, the confidence interval for $Y$ is given by

$$
\left[w_{1}, w_{2}\right] .
$$

The length of the statistical confidence interval is given by

$$
L\left(w_{1}, w_{2} \mid S_{k}\right)=S_{k}\left(w_{2}-w_{1}\right) \propto L\left(w_{1}, w_{2}\right)=\int_{w_{1}}^{w_{2}} \zeta(\tau) d \tau=\int_{w_{1}}^{w_{2}} \tau^{0} d \tau=w_{2}-w_{1} .
$$

In order to find the shortest length confidence interval $L\left(w_{1}, w_{2}\right)$, we should find a pair of decision variables $w_{1}$ and $w_{2}$ such that $L\left(w_{1}, w_{2}\right)$ is minimum.

Classical analytical approach: the problem statement in terms of the ancillary statistic $W$ and decision variables $w_{1}$ and $w_{2}$ :

Minimize

$$
L\left(w_{1}, w_{2}\right)=w_{2}-w_{1}
$$

subject to

$$
\int_{w_{1}}^{w_{2}} f(w) d w=\int_{w_{1}}^{w_{2}} \frac{k}{(1+w)^{k+1}} d w=F\left(w_{2}\right)-F\left(w_{1}\right)=\frac{1}{\left(1+w_{1}\right)^{k}}-\frac{1}{\left(1+w_{2}\right)^{k}}=1-\alpha .
$$

Differentiating $L\left(w_{1}, w_{2}\right)$ with respect to $w_{1}$, we get

$$
\frac{d L\left(w_{1}, w_{2}\right)}{d w_{1}}=\frac{d w_{2}}{d w_{1}}-1
$$

From (62) we find the derivative of $w_{2}$ with respect to $w_{1}$ as follows: 


$$
\frac{d}{d w_{1}} \int_{w_{1}}^{w_{2}} f(w) d w=\frac{d}{d w_{1}}(1-\alpha)
$$

that is

$$
f\left(w_{2}\right) \frac{d w_{2}}{d w_{1}}-f\left(w_{1}\right)=0
$$

Thus, we have

$$
\frac{d w_{2}}{d w_{1}}=\frac{f\left(w_{1}\right)}{f\left(w_{2}\right)}
$$

Letting this into (63), we get

$$
\frac{d L\left(w_{1}, w_{2}\right)}{d w_{1}}=\frac{f\left(w_{1}\right)}{f\left(w_{2}\right)}-1=\frac{\left(1+w_{2}\right)^{k+1}}{\left(1+w_{1}\right)^{k+1}}-1>0 .
$$

Optimal analytical results. It follows from (62) that

$$
1-\alpha<\frac{1}{\left(1+w_{1}\right)^{k}} \leq 1\left(\text { or } 0 \leq w_{1}<\left(\frac{1}{1-\alpha}\right)^{1 / k}-1\right),
$$

so that the minimum occurs at

$$
w_{1}=0, \quad w_{2}=\left(\frac{1}{\alpha}\right)^{1 / k}-1=1.154435 \text { (if } \alpha=0.1, k=3 \text { ). }
$$

The statistical shortest-length confidence interval for $Y$ based on $S_{k}$ is

$$
L\left(w_{1}, w_{2} \mid S_{k}\right)=S_{k} L\left(w_{1}, w_{2}\right)=S_{k}\left(\left(\frac{1}{\alpha}\right)^{1 / k}-1\right) .
$$

Note that the expected shortest-length confidence interval for $Y$ is given by

$$
E_{\beta}\left\{L\left(w_{1}, w_{2} \mid S_{k}\right)\right\}=E_{\beta}\left\{S_{k}\right\}\left(\left(\frac{1}{\alpha}\right)^{1 / k}-1\right),
$$

which is minimized, subject to (62).

The expected length of the interval that minimizes $E_{\beta}\left\{L\left(w_{1}, w_{2} \mid S_{k}\right)\right\}$ is which is also the expected length of the statistical shortest-length confidence interval $L\left(w_{1}, w_{2} \mid S_{k}\right)$ based on $S_{k}$. Note that the length of the interval $\left(0,\left(\frac{1}{\alpha}\right)^{1 / k}-1\right)$ goes to 0 as $k \rightarrow \infty$.

New simple computational method: the problem statement in terms of the decision variable $p$ (probability) and quantile functions $w_{1}$ and $w_{2}$ :

Minimize

$$
L^{2}\left(w_{1}, w_{2}\right)=\left(\int_{w_{1}}^{w_{2}} \zeta(\tau) d \tau\right)^{2}=\left(\int_{w_{1}}^{w_{2}} \tau^{0} d \tau\right)^{2}=\left(w_{2}-w_{1}\right)^{2}=\left(F^{-1}(1-\alpha+p)-F^{-1}(p)\right)^{2}=\left[\left(\frac{1}{\alpha-p}\right)^{1 / k}-\left(\frac{1}{1-p}\right)^{1 / k}\right]^{2},
$$

where the $p$-quantile function $w_{1}$ of $W$ is given by

$$
w_{1}=F^{-1}(p)=\left(\frac{1}{1-p}\right)^{1 / k}-1
$$


and the $(1-\alpha+p)$ - quantile function $w_{2}$ of $W$ is given by

$$
w_{2}=F^{-1}(1-\alpha+p)=\left(\frac{1}{\alpha-p}\right)^{1 / k}-1
$$

subject to

$$
0 \leq p \leq \alpha,
$$

Numerical solutions. The optimal numerical solution minimizing $L\left(w_{1}, w_{2}\right)$ can be obtained using the computer software "Solver". If, for example, $k=3, \alpha=0.1$, then the optimal numerical solution is given by

$$
p=0, \quad w_{1}=0, \quad w_{2}=1.154435
$$

with the $100(1-\alpha) \%$ shortest-length confidence interval

$$
L\left(w_{1}, w_{2}\right)=1.154435 .
$$

The $100(1-\alpha) \%$ equal tails confidence interval is given by

$$
L\left(w_{1}, w_{2} \mid p=\alpha / 2\right)=1.697174 .
$$

with

$$
p=0.05, \quad w_{1}=0.017245, \quad w_{2}=1.714418 .
$$

Inference. The proposed new simple computational method correctly recognized the adequate version of possible decision making and gave accurate numerical results.

Relative efficiency. The relative efficiency of $L\left(w_{1}, w_{2} \mid p=\alpha / 2\right)$ as compared with $L\left(w_{1}, w_{2}\right)$ is given by

$$
\text { rel.eff. } \cdot_{L}\left\{L\left(w_{1}, w_{2} \mid p=\alpha / 2\right), L\left(w_{1}, w_{2}\right)\right\}=\frac{L\left(w_{1}, w_{2}\right)}{L\left(w_{1}, w_{2} \mid p=\alpha / 2\right)}=\frac{1.154435}{1.697174}=0.68021 .
$$

\section{Cost-Effective Planning Unbiased Inspection Strategies}

In this paper we look at inspection strategies for items or structures that can be described as being in one of two states, one of which is preferable to the other. The inspection strategy defined is based on the conditional reliability of the structure. It is given as follows. Fix $0<\gamma<1$ and let

$$
\begin{gathered}
\tau_{1}=\arg \left(\operatorname{Pr}\left\{Y>\tau_{1}\right\}=\gamma\right), \\
\tau_{j}=\arg \left(\operatorname{Pr}\left\{Y>\tau_{j} \mid Y>\tau_{j-1}\right\}=\gamma\right), \quad j \geq 2,
\end{gathered}
$$

where $\left\{\tau_{j}\right\}_{j=1,2, \ldots}$ are inspection times, $Y$ is a random variable representing the lifetime of the component (structure). This is named as 'reliability-based inspection'. The value of $\gamma$ can be seen as 'minimum reliability required' (or 'reliability index'). If $F_{\beta}$, the structure lifetime distribution with the parameter $\beta$ is exponential (1), the definition of the inspection strategy (when the parameter $\beta$ is unknown) is equivalent to

$$
\tau_{j}=\arg \left(\bar{F}_{s_{k}}\left(\tau_{j}\right)=\gamma^{j}\right), \quad j \geq 1,
$$

where

$$
\bar{F}_{s_{k}}\left(\tau_{j}\right)=1-F_{s_{k}}\left(\tau_{j}\right) .
$$

If it is known that each inspection costs $c_{1}$ and the cost of leaving an undetected failure (some sort of 'damage') is $c_{2}$ per unit time, then the total expected cost per inspection cycle is given by

$$
C\left(\tau\left(s_{k}, \gamma\right)\right)=\sum_{j=1}^{\infty} \int_{\tau_{j-1}}^{\tau_{j}}\left[j c_{1}+c_{2}\left(\tau_{j}-y\right)\right] f_{s_{k}}(y) d y=c_{1} \sum_{j=1}^{\infty} j\left[F_{s_{k}}\left(\tau_{j}\right)-F_{s_{k}}\left(\tau_{j-1}\right)\right]+c_{2} \sum_{j=1}^{\infty} \tau_{j}\left[F_{s_{k}}\left(\tau_{j}\right)-F_{s_{k}}\left(\tau_{j-1}\right)\right]-c_{2} \int_{0}^{\infty} y f_{s_{k}}(y) d y
$$




$$
=c_{1} \sum_{j=0}^{\infty} \bar{F}_{s_{k}}\left(\tau_{j}\right)+c_{2} \sum_{j=1}^{\infty} \tau_{j}\left[\bar{F}_{s_{k}}\left(\tau_{j-1}\right)-\bar{F}_{s_{k}}\left(\tau_{j}\right)\right]-c_{2} E_{s_{k}}\{Y\}=c_{1}\left[\frac{1}{1-\gamma}+s_{k} \frac{c_{2}}{c_{1}}\left(\frac{1-\gamma^{1 / k}}{\gamma^{1 / k}-\gamma}-\frac{1}{k-1}\right)\right] .
$$

If, say, $c_{1}=1, c_{2}=2, s_{k}=50, k=3$, it follows from (85) that the reliability index rminimizing the total estimated cost per inspection cycle is given by

$$
\gamma^{*}=\arg \min _{0<\gamma<1} C\left(\tau\left(s_{k}, \gamma\right)\right)=0.825776
$$

where

$$
C\left(\tau\left(s_{k}, \gamma^{*}\right)=10.73406\right.
$$

The index of improvement percentage in effectiveness of the optimal inspection strategy (with $\gamma=\gamma^{*}=0.825776$ ) as compared with the standard inspection strategy (with $\gamma=\gamma_{\mathrm{st}}=0.95$ ) is given by

$$
I_{\text {imp.per. }}\left(\gamma^{*}, \gamma_{\text {st }}\right)=\frac{C\left(\tau\left(s_{k}, \gamma_{\text {st }}\right)\right)-C\left(\tau\left(s_{k}, \gamma^{*}\right)\right)}{C\left(\tau\left(s_{k}, \gamma_{\text {st }}\right)\right)} 100 \%=\frac{21.29704-10.73406}{21.29704} 100 \%=49.59835 \% \cong 50 \% \text {. }
$$

\section{Conclusion}

The new intelligent computational technique proposed in this paper represents the conceptually simple, efficient and useful method for constructing exact, optimal or improved statistical decision rules under parametric uncertainty of underlying models. This technique is based on the constructive use of the invariance principle in mathematical statistics. We have illustrated the technique for the exponential distribution. Applications to other log-location-scale distributions could follow directly. The methodology described here can be extended in several different directions to solve various problems arising in practice.

\section{References}

[1] Basu, D. (1977). On the elimination of nuisance parameters. Journal of the American Statistical Association, Vol. 72, 1977, pp. 355-366.

[2] Nechval, N. A. and Vasermanis, E. K. (2004). Improved Decisions in Statistics, Riga: Izglitibas soli, 2004.

[3] Nechval, N. A., Berzins, G., Purgailis, M., and Nechval, K. N. (2008). Improved estimation of state of stochastic systems via invariant embedding technique, WSEAS Transactions on Mathematics, Vol. 7, 2008, pp. 141-159.

[4] Nechval, N. A., Nechval, K. N., Danovich, V., and Liepins, T. (2011). Optimization of new-sample and within-sample prediction intervals for order statistics, in Proceedings of the 2011 World Congress in Computer Science, Computer Engineering, and Applied Computing, WORLDCOMP'11, Las Vegas Nevada, USA, CSREA Press, July 18-21, 2011, pp. 91-97.

[5] Nechval, N. A., Nechval, K. N., and Berzins, G. (2018). A new technique for intelligent constructing exact $\gamma$-content tolerance limits with expected $(1-\alpha)$-confidence on future outcomes in the Weibull case using complete or Type II censored data, Automatic Control and Computer Sciences (AC\&CS), Vol. 52, 2018, pp. 476-488.

[6] Nechval, N. A., Berzins, G., and Nechval, K. N. (2019). Intelligent technique of constructing exact statistical tolerance limits to predict future outcomes under parametric uncertainty for prognostics and health management of complex systems, International Journal of Advances in Computer Science \& Its Applications (IJCSIA), Vol. 9, 2019 , pp. 30-47.

[7] Nechval, N. A., Berzins, G., Nechval, K. N., and Krasts, J. (2019). A new technique of intelligent constructing unbiased prediction limits on future order statistics coming from an inverse Gaussian distribution under parametric uncertainty, Autom. Control Comput. Sci., Vol. 53, 2019, pp. 223-235.

[8] Nechval, N. A., Berzins, G., and Nechval, K. N. (2019). A novel intelligent technique for product acceptance process optimization on the basis of misclassification probability in the case of log-location-scale distributions, in: F. Wotawa et al. (Eds.), Advances and Trends in Artificial Intelligence. From Theory to Practice. IEA/AIE 2019, Lecture Notes in Computer Science, vol. 11606, 2019, pp. 801-818, Springer Nature Switzerland AG. 
[9] Nechval, N. A., Berzins, G., and Nechval, K. N. (2020). A novel intelligent technique of invariant statistical embedding and averaging via pivotal quantities for optimization or improvement of statistical decision rules under parametric uncertainty, WSEAS Transactions on Mathematics, vol. 19, pp. 17-38, 2020.

[10] Nechval, N. A., Berzins, G., Nechval, K. N., and Danovics, V. (2020). A novel technique for optimization of statistical decisions under parametric uncertainty through invariant statistical embedding and averaging in terms of pivotal quantities, Journal of Physics: Conference Series, Vol. 1603, 2020, p. 7, 012022.

[11] Nechval, N. A., Berzins, G., Nechval, K. N., and Danovics, V. (2020). Intelligent constructing optimal airline seat protection levels for multiple nested fare classes of single-leg flights, Journal of Physics: Conference Series, Vol. 1603, 2020, p. 7, 012023.

[12] Nechval, N. A. Berzins, G., and Nechval, K. N. (2020). A new technique of invariant statistical embedding and averaging via pivotal quantities for intelligent constructing efficient statistical decisions under parametric uncertainty, Automatic Control and Computer Sciences, vol. 54, 2020, pp. 191-206.

[13] Nechval, N. A. Berzins, G., and Nechval, K. N. (2020). Cost-effective planning reliability-based inspections of fatigued structures in the case of log-location-scale distributions of lifetime under parametric uncertainty, in Proceedings of the 30th European Safety and Reliability Conference and the 15th Probabilistic Safety Assessment and Management Conference, Edited by Piero Baraldi, Francesco Di Maio and Enrico Zio, ESREL2020-PSAM15, 1-6 November, 2020, Venice, Italy, pp. 455-462.

[14] Nechval, N. A. (2020). Intelligent constructing exact tolerance limits for prediction of future outcomes under parametric uncertainty, in Encyclopedia of Information Science and Technology, Fifth Edition (3 Volumes), USA, IGI Global, pp. 701-729, 2020.

[15] Nechval, N. A., Berzins, G., Nechval, K. N., and Tsaurkubule, Zh. (2021). A novel unified computational approach to constructing shortest-length or equal tails confidence intervals in terms of pivotal quantities and quantile functions, Automatic Control and Computer Sciences, Vol. 55, 2021, pp. 66-84.

[16] Nechval, N. A. Berzins, G., and Nechval, K. N. (2021). A new technique of invariant statistical embedding and averaging in terms of pivots for improvement of statistical decisions under parametric uncertainty, CSCE'20 - The 2020 World Congress in Computer Science, Computer Engineering, \& Applied Computing, July 27-30, 2020,Las Vegas, USA, in: H. R. Arabnia et al. (eds.), Advances in Parallel \& Distributed Processing, and Applications, Transactions on Computational Science and Computational Intelligence, pp. 257-274. Springer Nature Switzerland AG 2021.

[17] Nechval, N. A., Berzins, G., and Nechval, K. N. (2021). A new pivot-based approach to constructing prediction limits and shortest-length or equal tails confidence intervals for future outcomes under parametric uncertainty, Proceedings of the 31st European Safety and Reliability Conference (ESREL 2021), Edited by Bruno Castanier, Marko Cepin, David Bigaud, and Christophe Berenguer, 19-23 September 2021, Angers, France, pp. 2886-2893. Published by Research Publishing, Singapore, 2021.

[18] Nechval, N. A., Berzins, G., and Nechval, K. N. (2022). A new simple computational method of simultaneous constructing and comparing confidence intervals of shortest length and equal tails for making efficient decisions under parametric uncertainty. Proceedings of Sixth International Congress on Information and Communication Technology - ICICT 2021, Lecture Notes in Network and Systems (LNNS, volume 235), Yang X.-S., Sherratt S., Dey N., Joshi A. (eds), 25-26 February 2021, London, United Kingdom, pp. 473-482. Springer Nature Singapore 2022. 